\title{
Ocular Tissue Adhesive Application in DSAEK: a Comparative Study.
}

\author{
A.J. Kanellopoulos ${ }^{1,2} *$ and G. Asimellis ${ }^{1}$ \\ ${ }^{1}$ Laservision.gr Clinical and Research Eye Institute, Athens, Greece \\ ${ }^{2}$ Department of Ophthalmology, New York University Medical School, USA
}

Submission: October 30, 2015; Published: November 14, 2015

*Corresponding author: Anastasios John Kanellopoulos, Laservision.gr Eye Institute, 17 Tsocha Street, Athens, Greece, Postal Code: 11521, Tel: 30210 7472777; Fax: 30210 7472789; Email: ajk@brilliantvision.com

\begin{abstract}
Purpose

To evaluate clinical safety and efficacy of a novel use of an ocular tissue adhesive in Descemet's Stripping (Automated) Endothelial Keratoplasty (DSAEK).

Methods

35 consecutive DSAEK cases were evaluated retrospectively. In group- $\mathrm{A}\left(\mathrm{n}_{\mathrm{A}}=15\right)$ the tissue adhesive (Re Sure Adherent Ocular Bandage, Ocular Therapeutix, Inc., Bedford, MA) had been used, prior to placement of one suture, while in group- $\mathrm{B}\left(\mathrm{n}_{\mathrm{B}}=20\right)$, only nylon sutures were used for the closure of corneal incisions. Peri-operative complications were noted. Visual Acuity, refraction and topographic cylinder, Intraocular Pressure (IOP), and endothelial cell counts (ECC) were monitored long-term for up to two years.
\end{abstract}

\section{Results}

Follow-up time was $10.5 \pm 8.5$ ( 8 to 29 ) months. No case from group-A required any additional air insertion following the tissue adhesive application and no case required additional intra operative surgical manipulation for further graft centration. In group-B eighteen (out of twenty) cases required intra-operative supplemental air insertion, and four of those intra-operative repositioning of the graft. The differences in visual acuity and IOP were not statistically significant; ECC change of $-16 \%$ in group-A vs. $-21 \%$ was noted in group- B (statistically significant, $p=0.03$ ). Hyperopic shift was noted in both groups; cylinder reduction was noted, too, with group-A performing better.

\section{Conclusions}

Tissue adhesive may be a valuable adjunct in clear-cornea DSEAK by stabilizing the potential of air escape from the main incision inadvertently occurring during suture placement.

Keywords: LED Cassini; multi-color LED topography; Acanthamoeba keratitis; Point-source topography; Pentacam HR; Placido topography; Scheimpflug topometry; Differential topography; Irregular corneal astigmatism; Stray light measurements; C-Quant; Anterior-Segment Optical Coherence Tomography

\section{Introduction}

Descemet's Stripping (Automated) Endothelial Keratoplasty (DSAEK) surgery has become the standard of care worldwide for endothelial dysfunction management [1] superseding for this purpose Penetrating Keratoplasty (PK). PK involves a full corneal thickness, open-chamber procedure; among the disadvantages noted with this procedure are the prolonged visual rehabilitation, unpredictable cylindrical refractive changes (high postoperative astigmatism), susceptibility to ocular surface complications (wound dehiscence), and vulnerability to traumatic wound rupture [2,3]. DSAEK, introduced 2002, [4] involves the posterior cornea lamellae in a closed-chamber procedure [5]. Because of this, it is considered safer, provides faster visual recovery, [6] usually requires only few sutures and causes less astigmatic change,[7] overcoming some of the limitations of PK.

The corneal incisions associated with ocular corneal surgery, such as cataract and lamellar keratoplasty, are becoming smaller, depending on surgical instrumentation and techniques, as well 
as on implantation materials and designs. There is, nevertheless, concern that closure of DSAEK incisions with nylon sutures may induce air-bubble escape and possible graft slippage. Additionally, astigmatic changes may affect visual function. We have observed during our experience with DSAEK [8] topographic and tomographic changes consistent with significant irregular astigmatism along the incision site.

Application of ocular tissue adhesives for the closure of corneal incisions in DSAEK is considered a novel approach. This technique carries the benefits of an absence of risk of intraoperative needle stick injury and later suture removal. The purpose of this study was to evaluate the clinical efficacy of ocular tissue adhesive application in DSAEK cases.

\section{Materials and Methods}

This retrospective case series study received approval by the Ethics Committee of our Institution, adherent to the tenets of the Declaration of Helsinki. Informed written consent for the anonymous use of data had been obtained from each subject at the time of the first clinical visit or prior to the operation.

\section{Inclusion Criteria}

All consecutive DSEAK cases in our institution were considered for this study. The decision to proceed with ocular tissue adhesive-assisted closure (group- $A, n_{A}=15$ eyes) or traditional nylon-suture closure (group- $B, n_{B}=20$ eyes) or was based on random choice (coin toss). No case included in the study involved concurrent cataract removal and/or intraocular lens replacement surgery, which in our clinical practice corresponds to near $1 / 3$ of the DSAEK operations. All cases were performed by the same surgeon (AJK), as an ambulatory outpatient surgery procedure (not requiring hospital admission), and under monitored local anesthesia with peribulbar block [9].

\section{Surgical Technique}

We prepared the DSEAK grafts ourselves, with a Moria artificial chamber (Moria Surgical, Antony, France), an LSK (Gebauer Medizintechnik GmbH, Enzkreis, Germany) microkeratome (350 $\mu \mathrm{m}$ microkeratome head), and a Hanna suction punch block (Moria). Typical central graft thickness was of the order of $120 \mu \mathrm{m}$. A main clear cornea 4.50-mm incision at the 9th hour was employed for implantation using the singleuse Busin glide spatula \#17300 (Moria SA, Antony, France). Additionally, two clear cornea paracenteses were performed, a 1 -mm at the $6^{\text {th }}$ hour for a Moria coaxial microforceps forceps insertion and a $1-\mathrm{mm}$ at the $3^{\text {rd }}$ hour for the anterior chamber maintainer insertion and infusion. The DSEAK procedure was otherwise standard, to include scoring the host Descemet's with a reverse Sinskey hook, removal of the central hosts Descemet's membrane, and bi-manual pull of the DSEAK graft through the Busin glide spatula with forceps placed through the anterior chamber. Following the graft lenticule insertion in the anterior chamber, the anterior chamber maintainer infusion was restarted and the graft was unfolded. Last, a large air bubble was introduced in the anterior chamber in order to secure superior tamponade of the graft, against the host cornea. Following this last step, the two groups had different completion:

In group-A, the tissue adhesive (ReSure Adherent Ocular Bandage, Ocular Therapeutix, Inc., Bedford, MA) was mixed on the operating instrument stand, and applied in liquid form (painted) on the main incision borders with a special spear sponge following the absorption of any redundant surface fluid (Figure 1A). Within 3 to 5 seconds, the material was stable in soft form and any excess over the peripheral conjunctiva was removed with a dry spear sponge. The eye was observed for an additional 45 minutes for graft stability while the anterior chamber was filled with at least $75 \%$ with air.

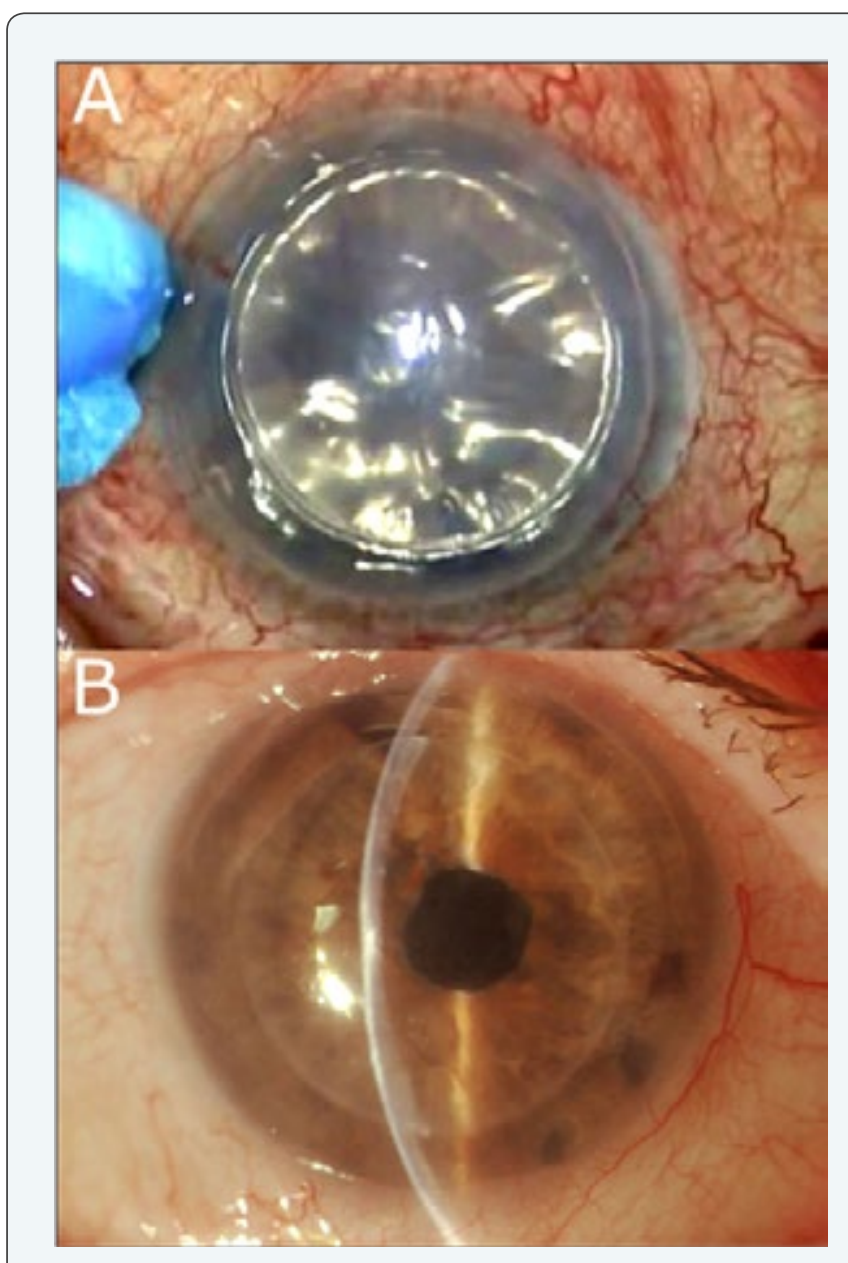

Figure 1:

A. Application of tissue adhesive in liquid form over the incision.

B. Slit-lamp picture of a sutureless DSEAK with the use of the tissue adhesive, 1 week post-operatively.

In group-B, following the DSEAK graft insertion, unfolding 


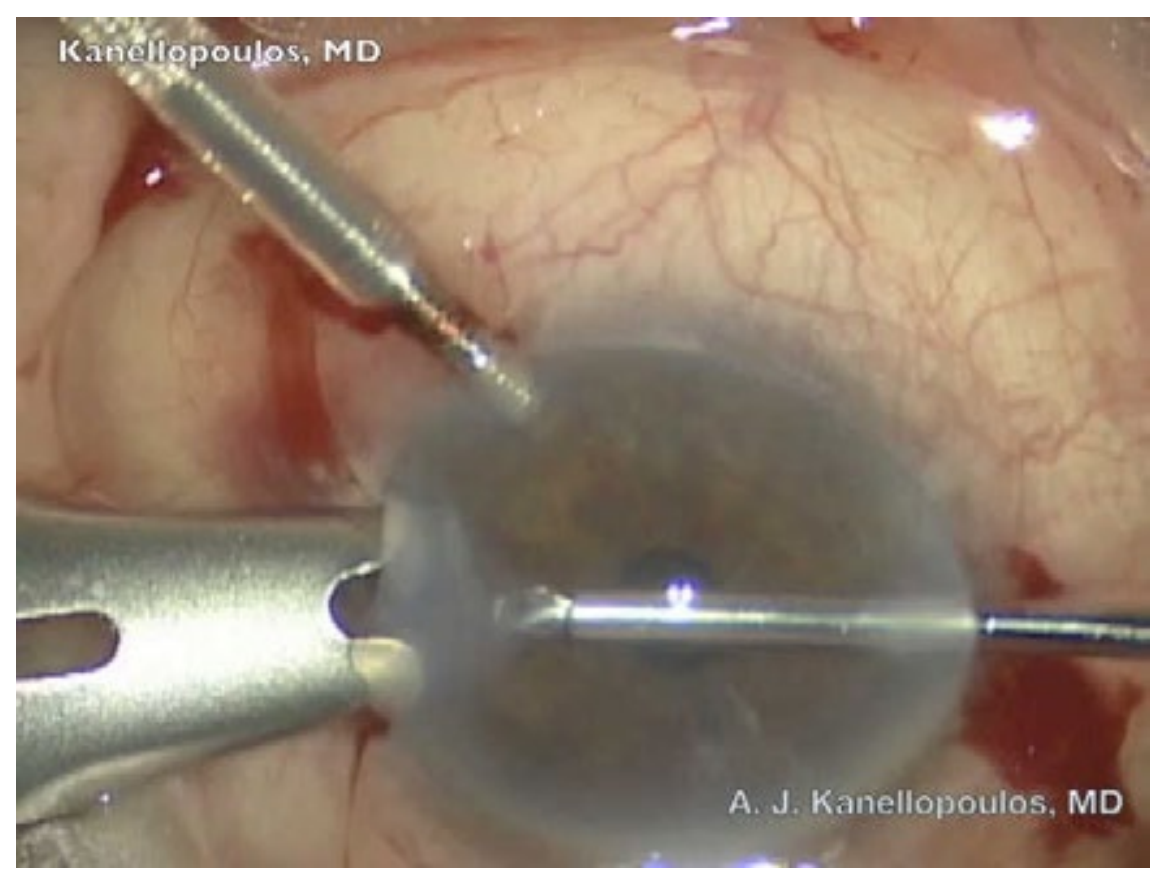

and air tamponade, only the 'matress' $10-0$ polypropylene sutures employing the CS 160-6 needle (Ethilon, Ethicon Inc, Somerville, NJ) were placed to secure the main incision. The sutures were removed typically at the one-month visit; in all cases the sutures had been removed well prior to the threemonth scheduled visit.

\section{Data Collection and Analysis}

All patients had been evaluated pre-operatively and at least one-year post-operatively for best-spectacle distance corrected visual acuity (CDVA) reported decimally, spherical and cylindrical error reported in diopters (D), Intraocular pressure (IOP) reported in $\mathrm{mmHg}$, and endothelial cell density (ECC) reported in cells $/ \mathrm{mm}^{2}$. In the case of pre-operative ECC, the data from the cornea bank certificates were used, while post-operatively, ECC was measured by specular microscopy (FA-3709, Konan Medical, Irvine, CA). Slit-lamp evaluation was also part of the complete ophthalmological evaluation performed. Figure 1B illustrates an example of slit lamp imaging from a sutureless DSEAK with the use of tissue adhesive, 1 week post-operatively.

Spherical and cylindrical error corresponds to the refraction for which the CDVA was reported, and was based on phoropter manifest refraction examination. IOP values were not adjusted for corneal thickness changes. In addition, qualitative evaluation by means of corneal tomography (Pentacam, Oculus Optikgeräte $\mathrm{GmbH}$, Wetzlar, Germany) and anterior-segment as well as retinal optical coherence tomography imaging (RtVue-100, Optovue, Fremont, CA) were part of the standard protocol performed during all visits [10]. Figure 2A presents an example of OCT imaging performed on DSAEK case two years postoperatively. Whenever possible, high-frequency scanning ultrasound imaging (Artemis ii+ superior, Artemis Medical Technologies Inc., Vancouver, British Columbia, Canada) was also employed for the imaging of the anterior segment. An example of Artemis cross-sectional imaging is illustrated in Figure 2B. Due to the nature of the condition, post-operative recovery was followed for at least once a year past their 12-month visit in all cases.

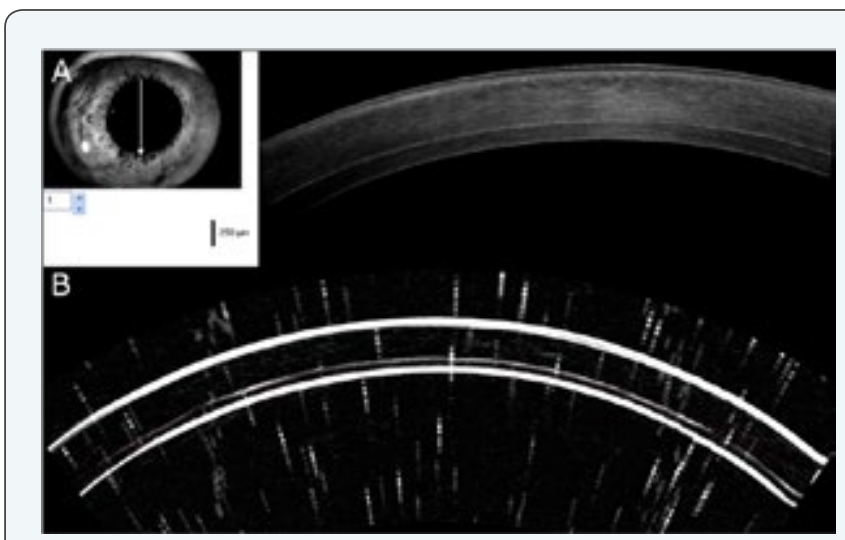

Figure 2: Cross-sectional imaging of a DSAEK case two years postoperatively: top, utilizing anterior-segment OCT, and bottom, utilizing high-frequency scanning ultrasound.

\section{Results}

The subject age in group- $\mathrm{A}\left(\mathrm{n}_{\mathrm{A}}=15,5\right.$ male and 10 female, 8 OD and 7 OS), at the time of the operation was $72.94 \pm 15.59$ (36 to 90$)$ years, while in group- $\mathrm{B}\left(\mathrm{n}_{\mathrm{B}}=20,6\right.$ male and 14 female, 9 OD and 11 OS) was $69.57 \pm 11.9$ (50 to 88 ) years.

No case from group-A required any additional intra operative air insertion following the tissue adhesive application and no 
case required additional intra operative surgical manipulation for further graft centration. No case required post-operative rebubbling or had any graft rejection incidence.

In group-B, 18/20 cases required intra-operative supplemental air insertion (re-bubbling), and four of those, intra-operative graft repositioning. Additionally, five cases required post-operative re-bubbling (on average 5.7 months post-operatively), and one case lead to graft rejection, followed by penetrating keratoplasty 9 months from the initial DSAEK operation. The cases from group-B with post-operative rebubbling or graft failure were excluded from the subsequent data analysis, leaving thus 14 cases whose refractive data are reported in this study, of which 4 were female and 11 male; 7 belonged to right eyes (OD) and 8 to left eyes (OS). Average follow-up time for all cases was $10.5 \pm 8.5$ (8 to 29) months.

In group-A preoperative CDVA was $0.14 \pm 0.17$ (0.01 to 0.60 ) decimal, spherical error was $-1.05 \pm 3.30(-9.50$ to +4.00$) \mathrm{D}$, cylinder was $-3.75 \pm 2.05$ ( -10.50 to -0.50$) \mathrm{D}$, IOP was $17.25 \pm 5.60$ (10 to 29 ) $\mathrm{mmHg}$, and graft/donor ECC was $2,567 \pm 310$ (1,790 to 2,935) cells $/ \mathrm{mm}^{2}$.

In group-B pre-operative CDVA was $0.15 \pm 0.16(0.01$ to 0.50$)$ decimal, spherical error was $-0.94 \pm 3.60(-12.50$ to +5.00$) \mathrm{D}$, cylinder was $-3.24 \pm 2.44(-10.00$ to -0.25$) \mathrm{D}$, IOP was $18.83 \pm 6.02$

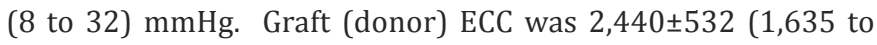
2,850 ) cells $/ \mathrm{mm}^{2}$.

Table 1 summarizes the pre-operative as well as the 3 -month and 12-month refractive and corneal data pertaining the two groups of study. The two groups were matched on all aspects of the parameters involved in the study (age, gender laterality, eye laterality, visual acuity, sphero cylindrical error, IOP and graft ECC), as none of the paired-test p-values was less than 0.05 .

The improvement in visual acuity within the same groups had a noted and statistically significant improvement at the 3-month interval $(\Delta=+0.22$ and +0.10 for group $-\mathrm{A}$ and $-\mathrm{B}$, respectively) as well as at the 12 -month interval $(\Delta=+0.31$ and +0.25$)$. IOP was increased $(\Delta=+5.51$ and $+4.65 \mathrm{mmHg}$ for group-A and $-\mathrm{B}$, respectively) at 3-months as well as at 12 -months $(\Delta=+5.77$ and $+5.22 \mathrm{mmHg}$ ). The increase in IOP between the two groups was rather similar, and not statistically significant. ECC change at 12 -months of $-16 \%$ was noted in group-A vs. $-21 \%$ in group-B (statistically significant difference between the two groups, $p$ $=0.03$ ).

Figure 3 illustrates the sphero cylindrical error (sphere and cylinder) pre-operatively, as well as 3 -months and at 12-months post-operatively. The spherical error indicated a hyperopic shift of $+1.97 \mathrm{D}$ in group-A and $+1.79 \mathrm{D}$ in group-B (not statistically significant difference between the two groups). Cylinder was improved (reduced), by $2.10 \mathrm{D}$ in group-A, and by $0.81 \mathrm{D}$ in group-B. The difference of cylinder improvement between the two groups was statistically significant $(p=0.012)$.

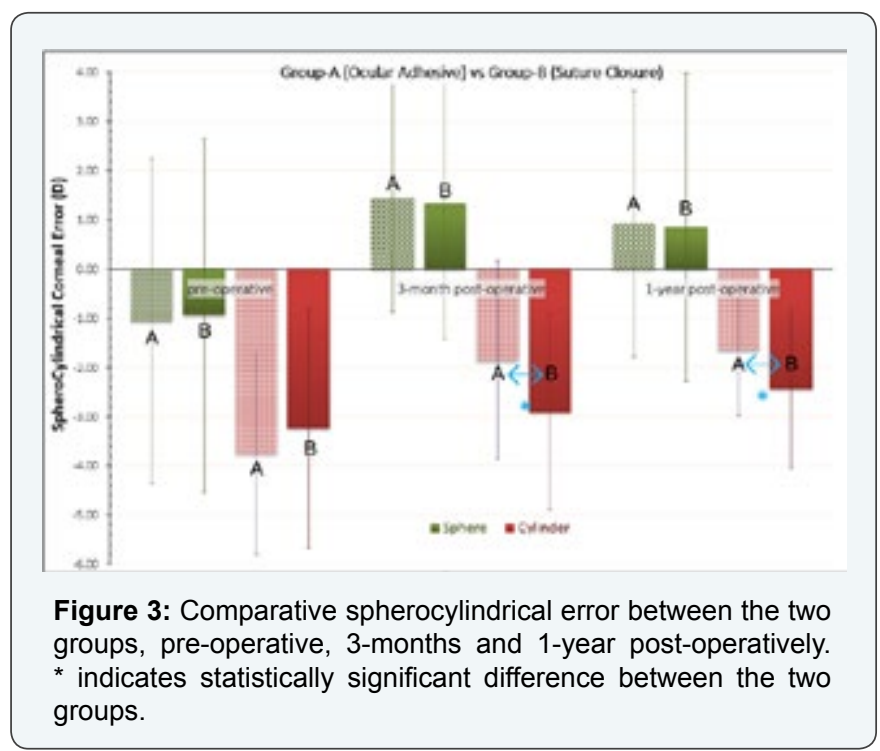

\section{Discussion}

Management of surgical cornea incisions with nylon sutures may have been the acceptable standard in the past, but there are a number of complications associated with this technique [11]. Induction of astigmatism, potential to fluid ingress and egress from the ocular surface,[12] endophthalmitis, [13] and increase of surgical time and patient discomfort are some that may be listed. There is, therefore, interest in an adhesive to replace and/or supplement sutures in the repair of corneal wounds and improve corneal incision sealing [14]. The material must be biocompatible, self-dissolving, and safe (eg without affecting visual function).

Cyanoacrylate, a biocompatible material, has long been employed in surgical incisions [15]. The latest cyanoacrylate adhesive for medical use, FDA-approved in 2002, was n-butyl-2cyanoacrylate (Indermil@, Vygon-Ecouen, Lansdale, PA). It has been used to close small skin wounds in pediatric patients, with successful results [16]. Applications in ocular surgery have also been reported $[17,18]$.

Polyethylene glycol (PEG) polymers are also among the materials considered for corneal wound sealing [19]. Biocompatible PEG polymers form the same type of hydrogel used in contact lenses in wetting agent applications [20]. The liquid hydrogel compound is administered (painted) over the wound, and polymerizes fast (in approximately half a minute) into a soft form that adheres to the ocular surface, forming a bandage that leads to a watertight seal. Two such ocular tissue adhesive products are the ReSure (Ocular Therapeutix, Inc., Bedford, MA) [21] and the OcuSeal (BD Medical, Waltham, MA) [22].

Ocular tissue adhesives have been evaluated for their 
applicability in limbal-conjunctival wound after fornix-based trabeculectomy [23] and cataract surgery [24]. To the best of our knowledge there is no report in the peer-reviewed literature on the topic of ocular tissue adhesives in DSAEK.

The present work is to the best of our knowledge the first investigation that presents the clinical applicability of employment of ocular tissue adhesive in DSAEK. We evaluated comparatively two matched groups of study over a long followup period. The differences in regard to far fewer cases needing intra-operative re-bubbling have been compelling. Postoperatively, similar visual acuity and IOP changes are noted. CDVA was improved in both groups, while also IOP increase has been noted; the latter can be explained by the thicker cornea (as a result of the DSAEK procedure) and the known dependence of IOP readings on central corneal thickness [25].

We noted a statistically significant ECC loss in both groups (by $-16 \%$ in group-A, and by $-21 \%$ in group-B). These data are in accordance with published results in the literature. For example, ECC loss of $-19 \%$ has been reported in DSAEK cases [26]. We note, however, that the ocular adhesive group-A appears to perform better in this aspect ( $p=0.045$ between the two groups).

Regarding the refractive data, both groups indicate a significant hyperopic shift. The average increase in sphere was more than +2.50 D at the 3-month interval and near +2.00 D at the 12-month interval. This hyperopic shift has been modeled, and the average predicted hyperopic shift in the overall power of the eye was calculated to be $+0.83 \mathrm{D}$ [27]. It is explained by the fact that the graft is thinner centrally, as a result of the microkeratome pass creation procedure over the donor cornea, and the known increased corneal thickness peripherally. It appears that our technique introduces more, but predictable hyperopic shift. As a result the graft is thinner centrally and thicker peripherally, the 'new' posterior corneal surface has a smaller radius of curvature; our calculations based on application of the Gullstrand's formula indicate that for approximately $1 \mathrm{~mm}$ change in the posterior curvature (e.g from 6.8 to $5.8 \mathrm{~mm}$ ) there is a corresponding 1.00 D change in the posterior corneal refractive power (e.g. from $-5.88 \mathrm{D}$ to -6.90$)$, resulting thus in $1.00 \mathrm{D}$ of hyperopic shift.

A noted improvement in cylinder was noted in both groups, with the ocular adhesive group-B appears to perform better in this aspect ( $\mathrm{p}=0.032$ ). The sutureless group-A had 12-month improvement in cylinder by $2.1 \mathrm{D}$, while the suture group-B by $0.8 \mathrm{D}$. This may be explained by the reduced effect on cornea distortion by the ocular adhesive. Considering the nonsymmetrical nature of the suture placement in the traditional wound sealing, the noted improvement in surgically-induced astigmatism in the sutureless group-A may offer perhaps the clinical advantage over the traditionally applied technique.

Application of a material that seals wounds safely, effectively, and comfortably enable better suture placement and possibly improve corneal surgical outcomes. This procedure enables better suture placement and provides the benefit of the reduction of wound leak during suturing and possible graft slippage. This procedure may also be applied to DMEK cases. Further studies involving the clinical impact of the use of these new polymer corneal sealants may be warranted.

\section{Conclusions}

This novel tissue adhes ive may be a valuable adjunct in sutureless DSEAK clear cornea surgery in enhancing intra operative anterior chamber stability and possibly offering more secure wound closure.

\section{References}

1. Lee WB, Jacobs DS, Musch DC, Kaufman SC, Reinhart WJ, et al. (2009) Descemet's stripping endothelial keratoplasty: safety and outcomes: a report by the American Academy of Ophthalmology. Ophthalmology 116(9):1818-1830.

2. Melles GR, Wijdh RH, Nieuwendaal CP (2004) A technique to excise the descemet membrane from a recipient cornea (descemetorhexis). Cornea 23(3): 286-288.

3. Price FW Jr, Price MO (2005) Descemet's stripping with endothelial keratoplasty in 50 eyes: a refractive neutral corneal transplant. J Refract Surg 21(4): 339-345.

4. Melles GR, Lander F, Rietveld FJ (2002) Transplantation of Descemet's membrane carrying viable endothelium through a small scleral incision. Cornea 21(4): 415-418.

5. Ghaznawi N, Chen ES (2010) Descemet's stripping automated endothelial keratoplasty: innovations in surgical technique. Curr Opin Ophthalmol 21(4): 283-287.

6. Maier P, Reinhard T, Cursiefen C (2013) Descemet stripping endothelial keratoplasty-rapid recovery of visual acuity. Dtsch Arztebl Int 110(21): 365-371.

7. Bahar I, Kaiserman I, Levinger E, Sansanayudh W, Slomovic AR, et al. (2009) Retrospective contralateral study comparing descemet stripping automated endothelial keratoplasty with penetrating keratoplasty. Cornea 28(5): 485-488.

8. Kanellopoulos AJ, Asimellis G (2014) Anterior-segment optical coherence tomography investigation of corneal deturgescence and epithelial remodeling after DSAEK. Cornea 33(4): 340-348.

9. Macaluso C (2008) Closed-chamber pulling-injection system for donor graft insertion in endothelial keratoplasty. J Cataract Refract Surg 34(3): 353-356.

10. Moutsouris K, Dapena I, Ham L, Balachandran C, Oellerich S, et al. (2011) Optical coherence tomography, Scheimpflug imaging, and slitlamp biomicroscopy in the early detection of graft detachment after Descemet membrane endothelial keratoplasty. Cornea 30(12): 13691375.

11. Hillier RJ, Ajit RR, Kelly SP (2009) Suture-related complications after cataract surgery: a patient safety issue. J Cataract Refract Surg 35(11): 2035-2036.

12. Masket S, Hovanesian JA, Levenson J, Tyson F, Flynn W, et al. (2014) Hydrogel sealant versus sutures to prevent fluid egress after cataract surgery. J Cataract Refract Surg 40(12): 2057-2066. 
13. Cooper MA, Holekamp NM, Bohigian G, Thompson PA (2003) Casecontrol study of endophthalmitis after cataract surgery comparing scleral tunnel and clear corneal wounds. Am J Ophthalmol 136(2):300305.

14. Grinstaff MW (2007) Designing hydrogel adhesives for corneal wound repair. Biomaterials 28(35): 5205-5214.

15. García Cerdá D, Ballester AM, Aliena-Valero A, Carabén-Redaño A Lloris JM, et al. (2014) Use of cyanoacrylate adhesives in general surgery. Surg Today 45(8): 939-956.

16. Schonauer F, Pereira J, La Rusca I, Harris J, Cullen K (2001) Use of Indermil tissue adhesive for closure of superficial skin lacerations in children. Minerva Chir 56(4): 427-429.

17. Sharma A, Mohan K, Sharma R, Nirankari VS (2013) Scleral Patch Graft Augmented Cyanoacrylate Tissue Adhesive for Treatment of Moderate-Sized Noninfectious Corneal Perforations (3.5-4.5 mm). Cornea 32(10): 1326-1330.

18. Gavin EA, Mahroo OA, Lim R, de Benito-Llopis L (2013) Using cyanoacrylate glue for corneal perforations: caution with use of a needle and syringe. Cornea 32(12): e193.

19. Strehin I, Ambrose WM, Schein O, Salahuddin A, Elisseeff J (2009) Synthesis and characterization of a chondroitin sulfate-polyethylene glycol corneal adhesive. J Cataract Refract Surg 35(3): 567-576.

20. Paterson SM, Liu L, Brook M, Sheardown H (2014) Poly(ethylene glycol)- or silicone-modified hyaluronan for contact lens wetting agent applications. J Biomed Mater Res A 103(8) :2602-2610.

21. Hirst LW (2013) Pterygium removal using a polyethylene glycol hydrogel adherent ocular bandage. Cornea 32(6): 803-805.

22. Uy HS, Kenyon KR (2013) Surgical outcomes after application of a liquid adhesive ocular bandage to clear corneal incisions during cataract surgery. J Cataract Refract Surg 39(11): 1668-1674.

23. Calladine D, Ratnarajan G, McAllister J (2011) New polyethylene glycol 'hydrogel bandage' technique to cover the conjunctival-limbal wound in fornix-based trabeculectomy surgery. Clin Experiment Ophthalmol 39: 921-923.

24. Kim T, Kharod BV (2007) Tissue adhesives in corneal cataract incisions. Curr Opin Ophthalmol 18: 39-43.

25. Grieshaber MC, Schoetzau A, Zawinka C, Flammer J, Orgul S (2007) Effect of central corneal thickness on dynamic contour tonometry and Goldmann applanation tonometry in primary open-angle glaucoma. Arch Ophthalmol 125(6): 740-744.

26. Gorovoy IR, Gorovoy MS (2015) Descemet Membrane Endothelial Keratoplasty Postoperative Year 1 Endothelial Cell Counts. Am J Ophthalmol 159: 597-600.

27. Hwang RY, Gauthier DJ, Wallace D, Afshari NA (2011) Refractive changes after descemet stripping endothelial keratoplasty: a simplified mathematical model. Invest Ophthalmol Vis Sci 52(2): 1043-1054. 